\title{
High-speed DD Transmission using a Silicon Receiver Co-integrated with a 28-nm CMOS Gain-tunable Fully-differential TIA
}

\author{
Yang Hong, Member, IEEE, Ke Li, Cosimo Lacava, Shenghao Liu, David J. Thomson, Fanfan Meng, \\ Xiaoke Ruan, Fan Zhang, Graham T. Reed, and Periklis Petropoulos, Fellow, OSA
}

\begin{abstract}
We report $>100-G b / s$ direct-detection (DD) transmission using an integrated silicon optical receiver, which comprised a fully-differential 28-nm complementary metal-oxide-semiconductor (CMOS) transimpedance amplifier (TIA) wire-bonded to a Silicon-Germanium (SiGe) balanced photodiode (PD). The fully-differential TIA architecture enabled significant bit-error-rate (BER) and signal-to-noise ratio improvements over a single-output TIA design when introducing it to the DD transmission. We experimentally validated its effectiveness in both back-to-back (B2B) and 2-km long standard single-mode fibre (SSMF) links using $50 / 100-G b / s$ signals, including Nyquist on-off keying (OOK), Nyquist 4-ary pulse amplitude modulation (PAM4), uniformly-loaded DD optical orthogonal frequency division multiplexing (DDO-OFDM) and adaptively-loaded DDO-OFDM. Furthermore, we demonstrate that the integrated optical receiver was capable of balancing the trade-off between its electrical bandwidth and transimpedance gain by simply adjusting a voltage supply to the TIA. Without the need for optical amplification and while keeping the BERs below the $7 \%$ forward error correction limit, up to $173.22-\mathrm{Gb} / \mathrm{s}$ and 139.86-Gb/s adaptively-loaded DDO-OFDM transmission was achieved for B2B and 2-km transmission, respectively. The demonstrated results highlight the potential of the integrated silicon optical receiver, fabricated using the standard CMOS process for high-speed and short-reach optical interconnects.
\end{abstract}

Manuscript was received XX xx, 2020, revised XX xx, 2020, accepted XX $\mathrm{xx}, 2020$. This work was supported by UK's EPSRC through the SPFS Programme (EP/L00044X/1), COALESCE project (EP/P003990/1) and juNIPeRS project (EP/T007303/1); and by National Natural Science Foundation of China (No. 61535002), and National Key R\&D Program of China (No. 2018YFB1801204). D.J. Thomson acknowledges funding from the Royal Society for his University Research Fellowship. K. Li acknowledges funding support from the State Key Laboratory of Advanced Optical Communication System and Networks, Peking University, China. (Corresponding author: Yang Hong).

Y. Hong, K. Li, C. Lacava, S. Liu, D.J. Thomson, F. Meng, G. T. Reed and P. Petropoulos are with the Optoelectronics Research Centre, University of Southampton, Southampton SO17 1BJ, UK. C. Lacava currently is with Department of Biomedical Engineering and Computer Science, University of Pavia, 27100 Pavia, Italy. (e-mails: y.hong@soton.ac.uk; kl@ecs.soton.ac.uk; cosimo.lacava@unipv.it; sl29g11@ecs.soton.ac.uk; d.thomson@soton.ac.uk; f.meng@soton.ac.uk; g.reed@soton.ac.uk; pp@orc.soton.ac.uk)

X. Ruan and F. Zhang are with State Key Laboratory of Advanced Optical Communication System and Networks, Frontiers Science Center for Nano-optoelectronics, Department of Electronics, Peking University, Beijing 100871. (e-mails: ruanxiaoke@pku.edu.cn; fzhang@pku.edu.cn)

Color versions of one or more of the figures in this paper are available online at http://ieeexplore.ieee.org.

Digital Object Identifier: XXXXXXX
Index Terms-Optical fibre communications; Direct-detection; Fully-differential TIA.

\section{INTRODUCTION}

$T$ HE annual global Internet Protocol traffic is predicted to reach 2.2 Zettabytes by 2020 , out of which more than $80 \%$ occurs in short-reach optical systems, especially in intra data centre interconnects (DCIs) [1-4]. In this regard, the specifications of $200-\mathrm{Gb} / \mathrm{s}$ and $400-\mathrm{Gb} / \mathrm{s}$ Ethernet have been standardised for DCIs with lengths of standard single-mode-fibre (SSMF) links ranging from $500 \mathrm{~m}$ to $10 \mathrm{~km}$ [5-6], highlighting the needs for $50 \mathrm{G} / 100 \mathrm{G}$ optical transceivers. To accommodate the need for high data rates whilst keeping the power consumption of short-reach optical interconnects as low as possible, energy-efficient and high bandwidth transceivers with small footprints are highly demanded. Owing to the beneficial properties of the complementary metal-oxide-semiconductor (CMOS) and silicon photonics technologies, such as their low cost, low power consumption and compact size, they are broadly considered as attractive routes for high-speed short-reach applications [7-8].

To this end, various $\mathrm{Si} / \mathrm{CMOS}$ transceiver solutions have been reported to achieve high-speed direct-detection (DD), which is currently the dominant solution for short-reach applications due to its simplicity and low cost [9-10]. In [11], an ultra-dense transceiver using an array of Germanium-Silicon (GeSi) electro-absorption modulators (EAMs) and photodiodes was demonstrated with the help of a multicore fibre. A $100-\mathrm{Gb} / \mathrm{s}$ real-time non-return-to-zero on-off keying (NRZ-OOK) link with a GeSi EAM and 130-nm SiGe BiCMOS-based transceiver chipsets was reported in [12], where up to 2-km long transmission over standard single-mode fibre (SSMF) was demonstrated to validate its potential for short-reach applications.

The transimpedance amplifier (TIA) typically included in optical receiver designs largely determines the overall sensitivity, operation speed, linearity performance and power consumption of the receiver module [13-17]. A number of optical receivers co-integrated with TIAs have been reported in the literature, the realisations of which were based on various technology platforms, including 250-nm, 130-nm \& 55-nm SiGe BiCMOS and 180-nm, 90-nm, 65-nm \& 28-nm CMOS 
[13-22]. Owing to the higher dynamic range and transition frequency of the BiCMOS technology [23-24], most of the high-speed demonstrations of TIA-integrated optical receivers to date have been realised in BiCMOS processes. For example, a $106-\mathrm{Gb} / \mathrm{s}$ optical receiver co-integrated with a 55-nm SiGe BiCMOS TIA was presented and investigated in a back-to-back (B2B) configuration in [17]. Compared to the BiCMOS technology, standard CMOS exhibits advantages such as lower cost and higher power efficiency [22]. Moreover, use of the same standard CMOS process for the TIA in the optical receiver as for the subsequent digital signal processing (DSP) chips [25] eliminates the need for interconnection between the two, which can then also share the same $\sim 1 \mathrm{~V}$ power supply. However, only a few works have reported high-speed transmission using a standard CMOS-based optical receiver. 32-Gb/s transmission using a mirrored-cascode differential TIA fabricated in 65-nm CMOS technology was reported in [18]. A 53-Gbit/s optical receiver co-integrated with a 28-nm CMOS TIA was demonstrated in [21], which achieved an energy efficiency of $0.65 \mathrm{pJ} / \mathrm{bit}$. Furthermore, a linear TIA in the 28-nm CMOS process was reported in [19], wherein standalone electrical measurements and simulations indicated that it was capable of operating beyond $100-\mathrm{Gb} / \mathrm{s}$ in B2B transmission. In [20], we have recently proposed a silicon optical receiver co-integrated with a fully-differential 28-nm standard CMOS TIA and demonstrated preliminary transmission results using the receiver module.

To investigate the full potential of the recently developed silicon optical receiver, in this work we present a comprehensive study of high-speed transmission in short-reach optical links using the integrated optical receiver and advanced DSP. The fully-differential TIA structure is shown to offer significant performance improvements compared to the single-ended TIA structure in both B2B and 2-km long SSMF links. Furthermore, in addition to single-carrier formats, which include $50-\mathrm{Gb} / \mathrm{s}$ Nyquist $\mathrm{OOK}$ and $100-\mathrm{Gb} / \mathrm{s}$ Nyquist 4-ary

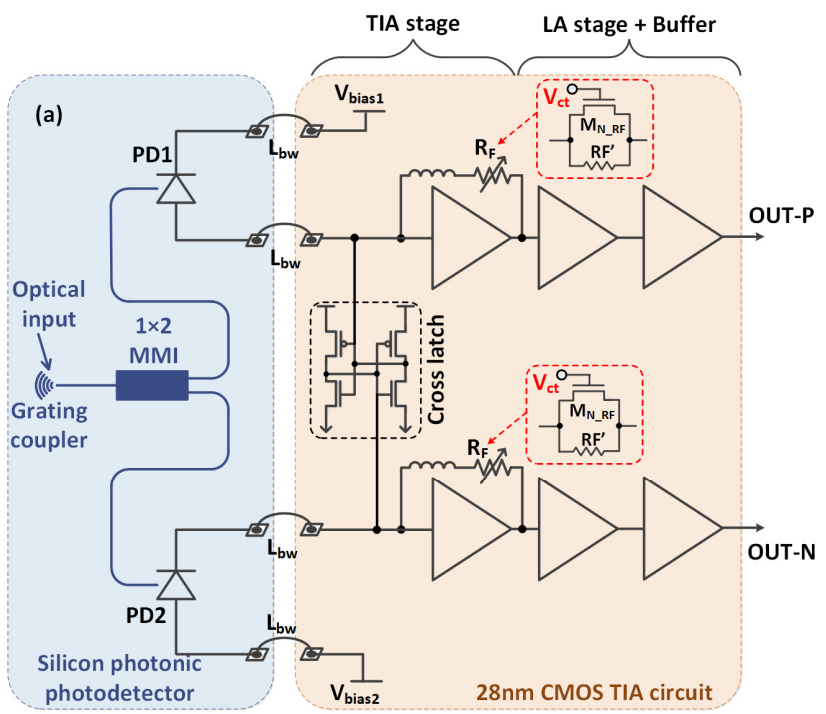

pulse amplitude modulation (PAM4), we also investigate the applicability of the receiver to multi-carrier DD optical orthogonal frequency division multiplexing (DDO-OFDM) with equivalent data rates, thereby assessing its linearity performance. Moreover, we demonstrate that the receiver module is capable of trading off transimpedance gain for electrical bandwidth, providing flexibility in maximising the system capacity for different reach applications.

The rest of this paper is structured as follows: in Section II, we will briefly describe the principle and implementation of the silicon optical receiver. Section III presents the experimental setup that was used to investigate the transmission performance of the receiver. Detailed experimental results as well as discussions are given in Section IV. Finally, Section V concludes this work.

\section{RECEIVER IMPLEMENTATION}

Fig. 1 shows the structure of the proposed optical receiver and microscope images of the top and side views of the fabricated silicon receiver. To balance factors, such as circuit architecture, device packaging, power efficiency, operation speed and footprint, a balanced photodetector (PD) and a 28-nm gain-tunable fully-differential TIA were synergistically co-designed in the integrated receiver. Note that while many of TIAs to date have been designed with a single-ended input followed by certain built-in single-to-differential conversion (e.g., [19]), here we used a SiGe balanced PD co-designed with the CMOS TIA to directly provide two fully differential outputs, as shown in Fig. 1(a). In this way, the extra single-to-differential conversion was avoided, and the circuit structure can also offer enhanced linearity.

The operational scheme of the integrated optical receiver is explained as follows: at the input of the receiver module, a grating coupler was used to couple the light signal into a $1 \times 2$ multimode interference (MMI) splitter which split its input into two identical parts. The resulting two optical signals were then
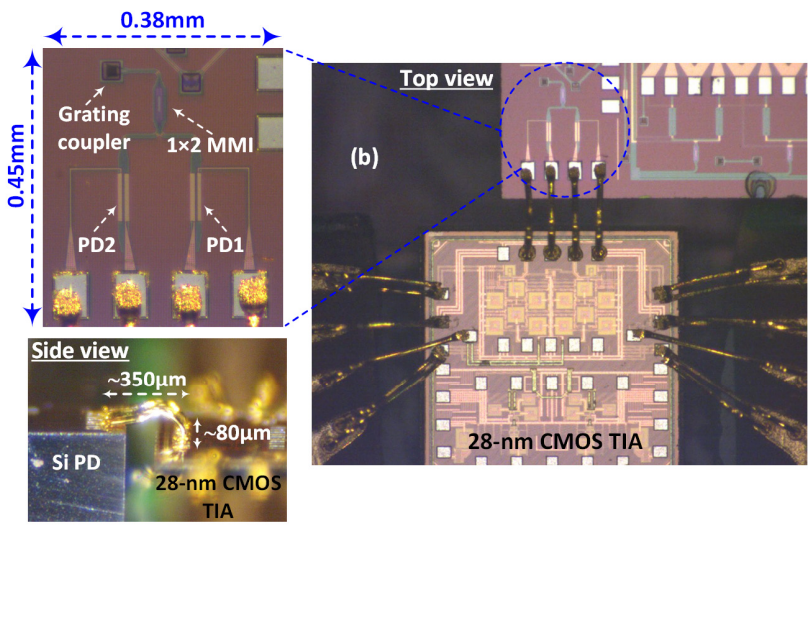

Fig. 1. (a) Block diagram of the proposed integrated optical receiver, (b) micro-scope views of the wire-bonding and the fabricated 28-nm CMOS TIA. 
fed into the balanced PD, wherein the cathode node of PD1 was tied to a bias voltage $V_{\text {bias } 1}=2 \mathrm{~V}$ whilst the anode of PD2 was tied to $\mathrm{V}_{\text {bias } 2}=-1 \mathrm{~V}$. Consequently, two complementary currents were generated in both direct and alternating domains. The SiGe balanced PD was fabricated via the IMEC ISIPP50G platform using the medium-bandwidth and highest-responsivity type (TE_GSLPINCFCWT). The 3-dB bandwidth of the PD was $\sim 33 \mathrm{GHz}$ whilst the responsivity at $1550 \mathrm{~nm}$ was around $1 \mathrm{~A} / \mathrm{W}$. Following the two complementary outputs of the balanced PD, the subsequent fully-differential TIA included three stages for PD1 and PD2, respectively, and the two arms were identical.

The first stage was designed based on the standard shunt feedback resistor topology, in which a N-type MOS (NMOS) transistor $\left(M_{N \_R F}\right.$ in Fig. 1(a)) was connected in parallel with a feedback resistor $\left(R F^{\prime}\right)$ to adjust the transimpedance gain of the TIA. This was simply achieved by tuning the voltage supply $\left(V_{c t}\right)$ to the two NMOS transistors. In principle, an increase in $V_{c t}$ results in a lower TIA gain, yet this in turn can lead to a larger electrical bandwidth of the integrated receiver. We will show in the following sections that this gain/bandwidth-tunable feature is particularly beneficial for short-reach IM/DD fibre systems, in which the chromatic dispersion (CD) induced power fading highly depends on the link length. The second stage was the CMOS-based limiting amplifier, whereas the third stage served as the 50 -ohm output buffer. Although the designed receiver only supports a single polarization (due to the use of the grating coupler and MMI splitter), it is feasible to combine it with techniques that address the polarization diversity issue in silicon receivers [26-27], so that an optical signal can be coupled into the receiver regardless of its polarization state.

Some dimensional information on the physical implementation of the balanced PD module is shown in Fig.1(b). The wire-bonding to the 28-nm CMOS TIA module is also shown in the figure. With a $1-\mathrm{V}$ supply, the whole TIA circuit including the output buffer drew a current of $\sim 51.4 \mathrm{~mA}$. Simulations showed that the transimpedance gain and the $3-\mathrm{dB}$ bandwidth of the integrated optical receiver were about 52.5 $\mathrm{dB} \Omega$ and $20 \mathrm{GHz}$, respectively. It is worth noting that the there is a trade-off between receiver bandwidth and transimpedance gain (and hence noise performance) for the TIA-integrated receiver. For our proposed receiver that operates under a standard digital supply voltage $(\sim 1 \mathrm{~V})$, we have chosen the optimal gain-bandwidth product, which yielded an overall receiver bandwidth of around $20 \mathrm{GHz}$ [22]. The properties of the fabricated optical receiver have been reported in detail in [22]. In the experiments conducted in this work, we study the transmission performance achieved using the optical receiver with single/differential output and assess its gain-tuning capability.

\section{EXPERIMENTAL SETUP}

Fig. 2(a) shows the experimental setup used to evaluate the transmission performance of the integrated optical receiver described in Section II. A continuous wave (CW) laser operating at $1550 \mathrm{~nm}$ was used as the optical carrier. The output power of the $\mathrm{CW}$ laser was $16 \mathrm{dBm}$. The offline-generated signals were fed into an arbitrary waveform generator (AWG) for digital-to-analog conversion, and the resulting analog signals were first amplified by an electrical amplifier (EA) before being used to modulate a $\mathrm{LiNbO}_{3}$ Mach-Zehnder Modulator (MZM). In the experiments, we evaluated the transmission performance either in B2B or after transmission in a 2-km long length of SSMF. At the receiver, a polarisation controller (PC) was used to align the polarisation of the input optical signal with that of the grating coupler integrated in the silicon optical receiver (TE polarization). A variable optical attenuator was adopted after the PC to adjust the received optical power (ROP), wherein $99 \%$ was launched into the integrated receiver module whilst the remaining $1 \%$ was used for ROP monitoring. Note that the ROP in this work refers to the optical power to the overall integrated receiver, therefore the optical power to each of the two PDs (after the 3-dB MMI splitter) in the single-ended and differential cases was identical. However, the sum optical power in the differential-output case was double that of the single-ended case. The two differential outputs of the integrated receiver were captured by an $80-\mathrm{GSa} / \mathrm{s}$ digital storage oscilloscope (DSO) for further offline DSP. To operate the DSO in the high-sampling-rate (high-bandwidth) mode, the two differential outputs were recorded by two separate channels of the DSO one by one. For all modulation formats, only one of the DSO's captured signals (OUT-P) was used for synchronization in the single-ended case, whereas in the differential-output case, to jointly process the two receiver outputs, synchronization was first implemented for the captured signals. Thereafter, the two synchronized signals were subtracted from one another. The resulting signal was then processed by further DSP, which was identical to that used in the single-ended case.

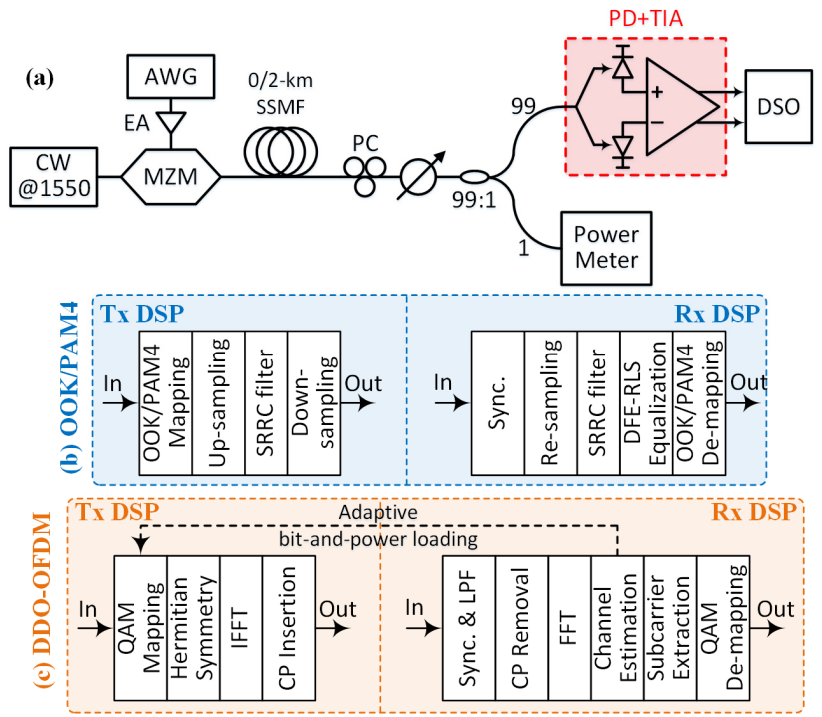

Fig. 2. (a) Experimental setup, (b) block diagram of the DSP for Nyquist OOK/PAM4, and (c) block diagram of the DSP for DDO-OFDM.

We experimented both with single-carrier (50-GBaud Nyquist OOK/PAM4) and multi-carrier (DDO-OFDM) 
formats. The DSP blocks for the processing of the single-carrier signals are shown in Fig. 2(b). Initially, the binary input at the transmitter was mapped to OOK/PAM4 symbols. The mapped signal was then up-sampled to perform Nyquist filtering using a square-root-raised-cosine filter (SRRC) with a roll-off factor of 0.1 . Subsequently, the filtered signal was down-sampled and fed into the AWG. The sampling rate of the AWG was fixed at $90-\mathrm{GSa} / \mathrm{s}$ for both the $50-\mathrm{Gb} / \mathrm{s}$ Nyquist $\mathrm{OOK}$ and the $100-\mathrm{Gb} / \mathrm{s}$ PAM4 signals. At the receiver, the DSP for the synchronised signal included re-sampling, matched SRRC filtering and a decision-feedback equalizer (DFE) using the recursive least squares (RLS) algorithm. We used 17 feedforward and 7 feedback taps for the T/2-spaced DFE. The forgetting factor used in the RLS algorithm was 0.99. After equalization, OOK/PAM4 de-mapping was performed to obtain the binary bits, which were subsequently used for bit error rate (BER) calculation via error counting.

Fig. 2(c) shows the offline DSP of DDO-OFDM. For the uniformly-loaded DDO-OFDM, the input binary bits were mapped to either 4-ary quadrature amplitude modulation (4QAM) or 16QAM symbols. After QAM mapping, Hermitian extension was applied, so that the generated signals after the inverse fast Fourier transform (IFFT) were real-valued. The block size of the IFFT was 512. Then, a cyclic prefix (CP) of $1 / 16$ of the length of one DDO-OFDM symbol was inserted in front of each DDO-OFDM symbol. The resulting time-domain waveform was fed into the AWG, using a sampling rate of 70 $\mathrm{GSa} / \mathrm{s}$. To maintain a similar signal bandwidth as in the Nyquist OOK/PAM4 cases (i.e. $25 \mathrm{GHz}$ ), the number of modulated data subcarriers was kept at 183 , which corresponded to an electrical bandwidth of $\sim 25.02 \mathrm{GHz}$. Accordingly, the data rates of 4QAM- and 16QAM-based DDO-OFDM were $\sim 50.04 \mathrm{~Gb} / \mathrm{s}$ and $\sim 100.08 \mathrm{~Gb} / \mathrm{s}$, respectively. At the receiver, the synchronised data was first low-pass filtered (LPF). After that, CP removal, FFT, channel estimation (one-tap equalization), data subcarrier extraction and QAM de-mapping were conducted to recover the binary bits. As with the Nyquist OOK/PAM4 formats, the BER of the DDO-OFDM transmission was also obtained via error counting. For the adaptively-loaded DDO-OFDM format, the estimated signal-to-noise ratios (SNRs) of data subcarriers were used for adaptive bit-and-power loading in the QAM mapping, as indicated in Fig. 2(c). Note that we adopted the following two different modes when performing adaptive loading [28-29]: (1) for the scenario where the aggregate data rate was fixed (i.e., $\sim 50 \mathrm{~Gb} / \mathrm{s}$ or $\sim 100 \mathrm{~Gb} / \mathrm{s}$ ), the objective of the adaptive loading was to minimise the BER of the transmission for the specified data rate; and (2) for the scenario that capacity maximization was targeted, the adaptive loading aimed at maximising the achievable data rate whilst keeping the BER of the transmission below the $7 \%$ forward error correction (FEC) limit, i.e., $\mathrm{BER} \leq 3.8 \times 10^{-3}$.

\section{EXPERIMENTAL RESULTS}

In this section, we present the transmission results obtained for the different modulation formats. We first investigate the performance of $50-/ 100-\mathrm{Gb} / \mathrm{s}$ single-carrier and multi-carrier formats in the silicon optical receiver-based transmission system, where $V_{c t}$ was fixed at $1.05 \mathrm{~V}$. A detailed performance comparison is then provided. Finally, the capacity maximization via the adaptively-loaded DDO-OFDM is presented for both B2B and 2-km long transmission in SSMF under various $V_{c t}$ settings.

\section{A. 50-/100-Gb/s single-carrier Nyquist OOK/PAM4}

Fig. 3 shows the BER and SNR versus ROP of 50-Gb/s Nyquist OOK transmission in the $\mathrm{B} 2 \mathrm{~B}$ link. We note that the actual ROP to each PD was around 9-dB lower, due to $\sim 6-\mathrm{dB}$ coupling loss to the optical receiver and the 3 -dB splitting in the $1 \times 2$ MMI. It is observed that the fully-differential TIA structure offered a significant performance improvement relative to the single-ended output. Specifically, a ROP of $\sim-9 \mathrm{dBm}$ was required to achieve a BER below the 7\% FEC limit when using the differential structure, as compared to $\sim-7.5 \mathrm{dBm}$ for the case of the single-ended output. A 3-dB higher SNR relative to the single-ended case was obtained. For reference, the recovered eye diagrams for the two cases at $-9 \mathrm{dBm}$ are also given in Fig. 3 . The BERs of the cases represented in these diagrams were $3.65 \times 10^{-2}$ and $3.66 \times 10^{-3}$, respectively.

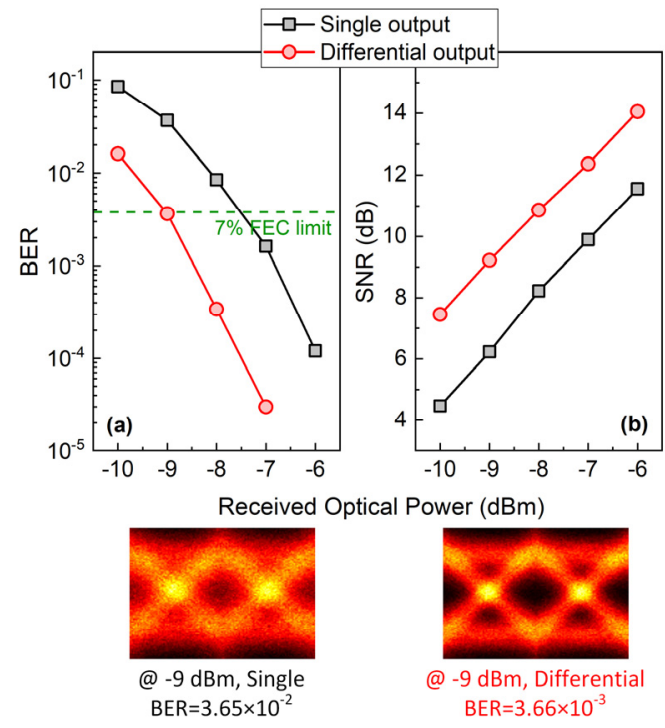

Fig. 3. Results of $50-\mathrm{Gb} / \mathrm{s}$ Nyquist OOK transmission in the B2B link.

The same signal was transmitted over the 2-km long SSMF and the corresponding BER and SNR results are shown in Fig. 4. As before, operating the receiver with a differential output resulted in a 3-dB SNR improvement, which was also confirmed in the BER results: a $\sim-7.5-\mathrm{dBm}$ ROP was required for a BER below the 7\% FEC limit, as opposed to $-6 \mathrm{dBm}$ in the single-ended case. 


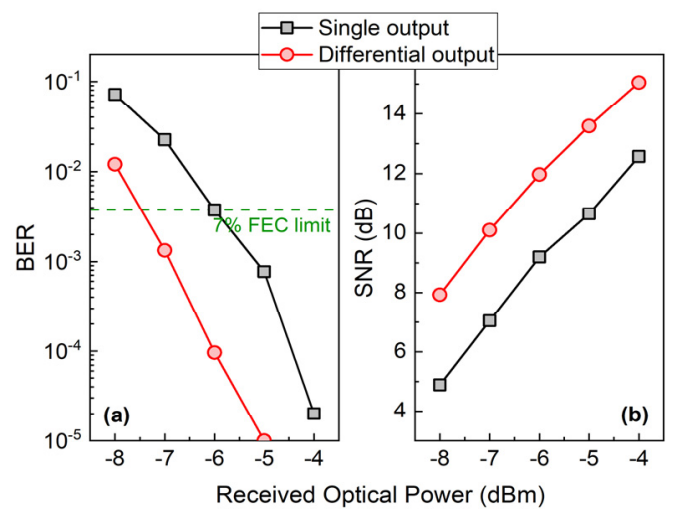

Fig. 4. Results of 50-Gb/s Nyquist OOK transmission in the 2-km length SSMF link.

Similar conclusions were drawn from the Nyquist PAM4 transmission experiments (see Figs. 5 and 6 for the B2B and 2-km SSMF transmission, respectively). Note that the same baud rate was used as before, therefore the transmission rate was doubled in this case to $100 \mathrm{Gbit} / \mathrm{s}$. For reference, the ROP to achieve a BER at the $7 \%$ FEC limit was $\sim-4 \mathrm{dBm}$ and $-1.6 \mathrm{dBm}$ for the differential and single-ended cases for the $\mathrm{B} 2 \mathrm{~B}$ link, respectively. The corresponding values after $2 \mathrm{~km}$ transmission were increased to $-2 \mathrm{dBm}$ and $0.1 \mathrm{dBm}$, respectively. The eye diagrams of the recovered PAM4 signals shown in Fig. 5 were obtained at a ROP of $-4 \mathrm{dBm}$. The corresponding BERs for the single-ended and differential outputs at these power levels were $2.25 \times 10^{-2}$ and $3.39 \times 10^{-3}$, respectively.

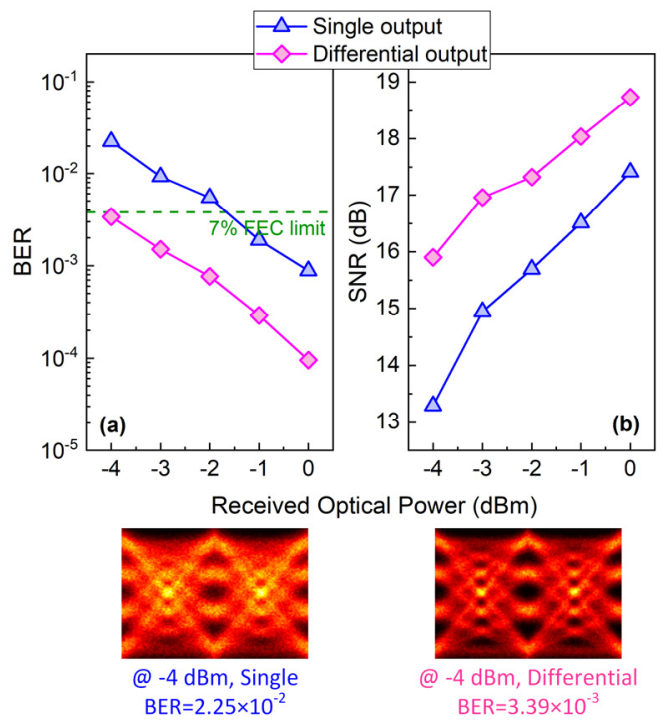

Fig. 5. Results of 100-Gb/s Nyquist PAM4 transmission in the B2B link.

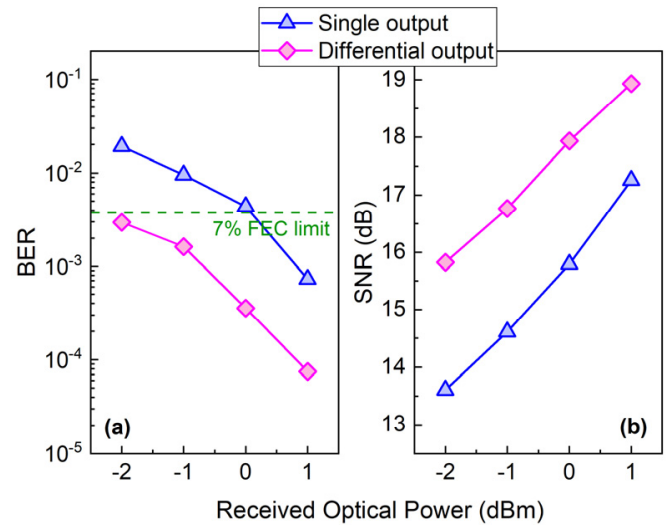

Fig. 6. Results of 100-Gb/s Nyquist PAM4 transmission in the 2-km length SSMF link.

\section{B. 50-/100-Gb/s multi-carrier DDO-OFDM}

In this sub-section, we present the results obtained by using the multi-carrier DDO-OFDM format, with and without adaptive loading. Compared to the single-carrier OOK/PAM4 formats, the time-domain signal of multi-carrier DDO-OFDM exhibits a much higher peak-to-average power ratio (PAPR). Therefore, it is more sensitive to the nonlinearities induced both during transmission and by the receiver. However, it offers enhanced flexibility, especially when targeting a high data rate in a given bandwidth, since both the number of bits per symbol and the power can be adaptively allocated to data subcarriers for performance optimization. To make a fair comparison to the single-carrier OOK/PAM4 results presented in Section IV.A, we also adopted a $\sim 25-\mathrm{GHz}$ signal bandwidth, which is comparable to that of the OOK/PAM4 transmission. 4QAM and 16QAM were used as the QAM formats to realise $\sim 50-\mathrm{Gb} / \mathrm{s}$ and $\sim 100-\mathrm{Gb} / \mathrm{s}$ uniformly-loaded DDO-OFDM transmission, respectively.

Fig. 7 shows the results of the 50-Gb/s 4QAM DDO-OFDM $\mathrm{B} 2 \mathrm{~B}$ tests. Once again, the differential output structure provides significant BER reduction and SNR improvement. At a ROP of $-5 \mathrm{dBm}$, the BER of the transmission can be reduced from $2.09 \times 10^{-2}$ to $1.95 \times 10^{-3}$, which corresponded to a $\sim 3-\mathrm{dB}$ SNR improvement, as shown in Fig. 7(b). For reference, the corresponding recovered 4QAM constellations at $-5-\mathrm{dBm}$ ROP are also shown in Fig. 7. The results obtained after transmission over 2-km of SSMF are shown in Fig. 8. It is shown that ROPs of around $-0.8 \mathrm{dBm}$ and $-2.8 \mathrm{dBm}$ were required to achieve a BER below the $7 \%$ FEC limit for the single- and differential-output cases, respectively. Compared to the B2B case, the transmission in the $2-\mathrm{km}$ length of SSMF induced more than $2.5-\mathrm{dB}$ sensitivity penalty. 


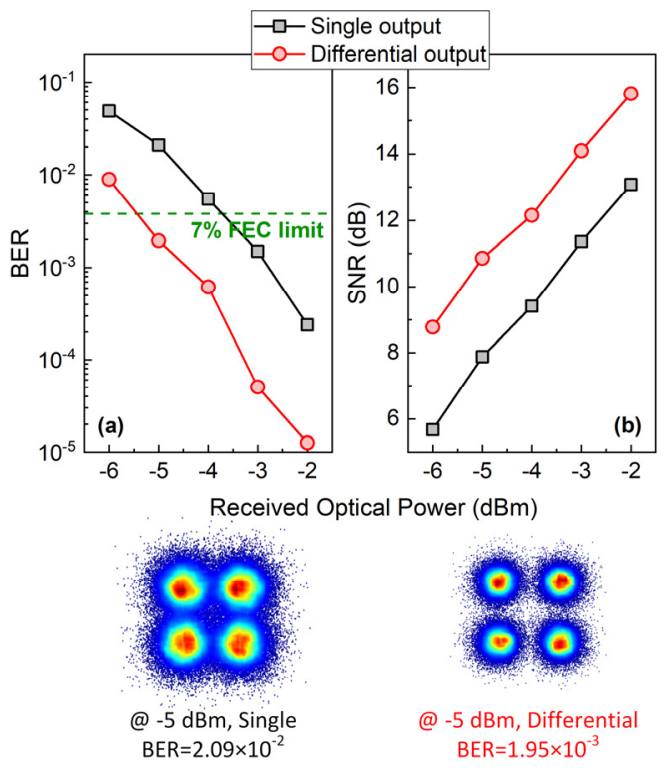

Fig. 7. BER and SNR results of the $50-\mathrm{Gb} / \mathrm{s} 4 \mathrm{QAM}$ DDO-OFDM transmission in the B2B link.

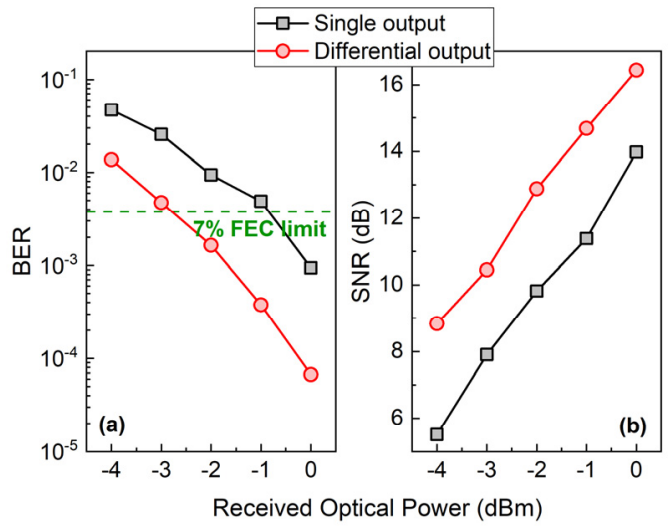

Fig. 8. BER and SNR results of the 50-Gb/s 4QAM DDO-OFDM transmission in the 2-km length of SSMF link.

We also conducted transmission of $100-\mathrm{Gb} / \mathrm{s}$ DDO-OFDM by doubling the order of the QAM format to 16QAM. The BER and SNR results of B2B and the 2-km long link are shown in Fig. 9 and Fig. 10, respectively. The conclusions from these measurements are consistent to the previous cases. As anticipated, a higher data rate was achieved at the expense of an increased required ROP for the same BER performance. Indicatively, around $3-\mathrm{dBm} \mathrm{ROP}$ was required in the differential output case, in order to achieve a BER at the $7 \%$ FEC limit after transmission in 2-km long SSMF, as shown in Fig. 10.

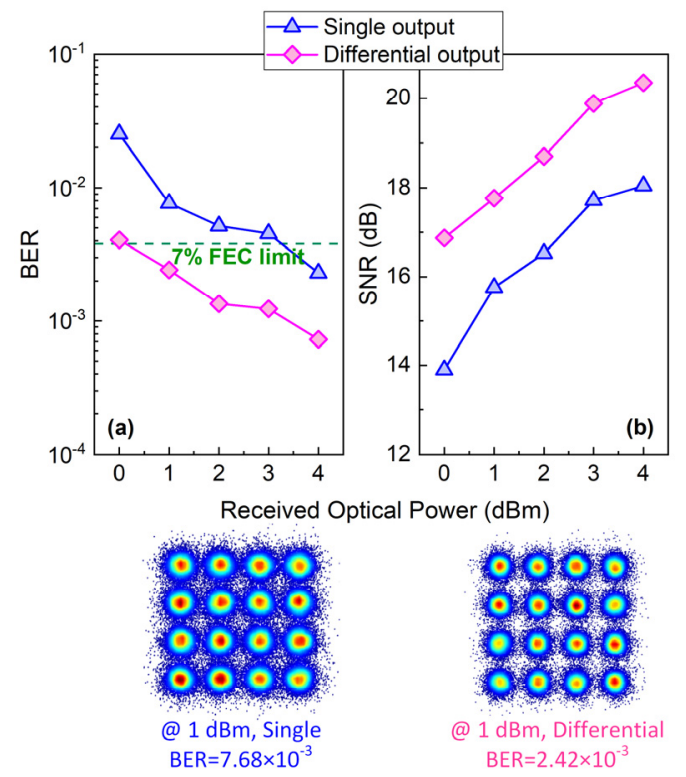

Fig. 9. BER and SNR results of the 100-Gb/s 16QAM DDO-OFDM transmission in the B2B link.

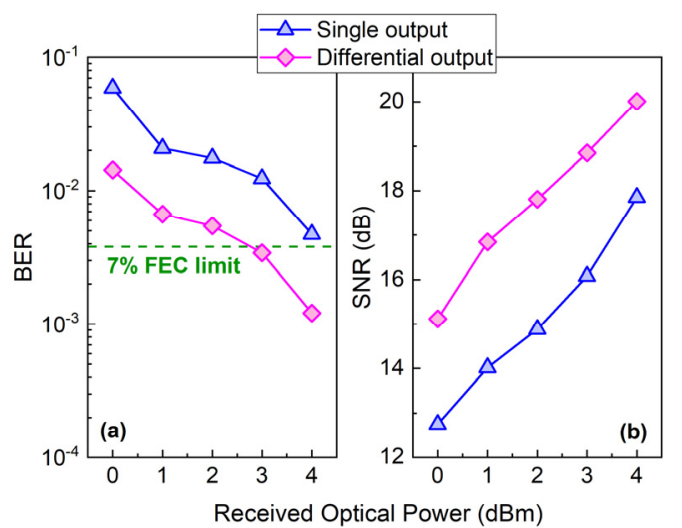

Fig. 10. BER and SNR results of the 100-Gb/s 16QAM DDO-OFDM transmission in the 2-km length of SSMF link.

We next experimented with adaptive bit-and-power-loaded DDO-OFDM, in order to take advantage of the multi-carrier nature of the signal, which enables either optimising its BER performance or improving the capacity of the system. We initially maintained the total data rate to either $50 \mathrm{~Gb} / \mathrm{s}$ or 100 $\mathrm{Gb} / \mathrm{s}$ and measured the receiver sensitivity. Note that the objective of this set of measurements was to minimise the BER under the fixed data rates of $50 \mathrm{~Gb} / \mathrm{s}$ or $100 \mathrm{~Gb} / \mathrm{s}$. Therefore, an insufficient ROP may indeed result in a BER higher than the $7 \%$ FEC limit (in contrast to the scenario considered in sub-section IV-D below)._Figs. 11 and 12 outline the BER results obtained at $\mathrm{B} 2 \mathrm{~B}$ and after $2-\mathrm{km}$ transmission, respectively. In all cases, the differential TIA structure offered around 2-dB ROP sensitivity improvement at a BER threshold of $3.8 \times 10^{-3}$. Around 5-dB additional power was required at the receiver when doubling the data rate from $50 \mathrm{~Gb} / \mathrm{s}$ to $100 \mathrm{~Gb} / \mathrm{s}$, in order to maintain a similar BER performance. 


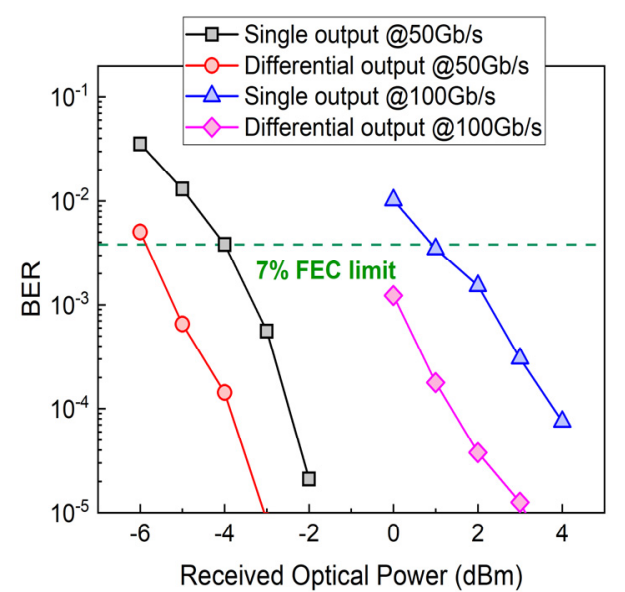

Fig. 11. BER versus ROP of $50-\mathrm{Gb} / \mathrm{s}$ and $100-\mathrm{Gb} / \mathrm{s}$ adaptively-loaded DDO-OFDM in the B2B link.

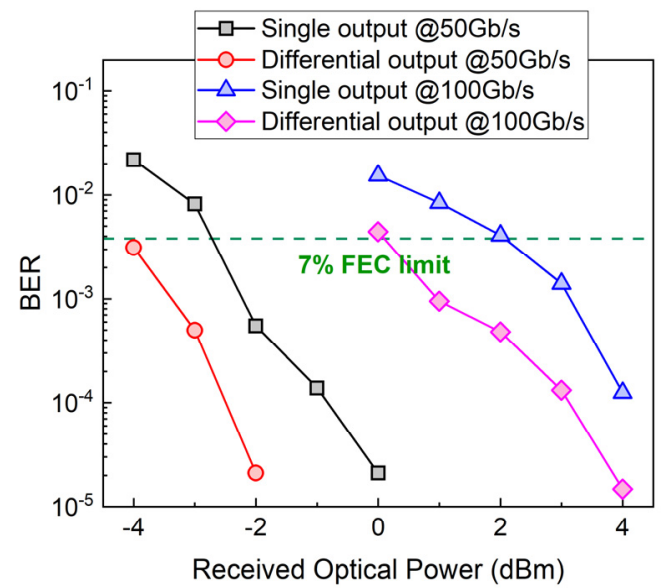

Fig. 12. BER versus ROP of $50-\mathrm{Gb} / \mathrm{s}$ and $100-\mathrm{Gb} / \mathrm{s}$ adaptively-loaded DDO-OFDM in the 2-km length of SSMF link.

\section{Comparison between the single-and multi-carrier formats}

In this sub-section, we analyse the performance of the silicon receiver when used in either differential or single-ended output modes for the various modulation formats considered in the experiments. To facilitate the discussion, we summarise in Tables I and II the ROP requirements (for achieving a BER at the $7 \%$ FEC limit) for $50-\mathrm{Gb} / \mathrm{s}$ and $100-\mathrm{Gb} / \mathrm{s}$ data rates both in the $\mathrm{B} 2 \mathrm{~B}$ and the 2-km transmission cases. It can be easily concluded that when using a $25-\mathrm{GHz}$ bandwidth and transmitting at $50 \mathrm{~Gb} / \mathrm{s}$, the single-carrier Nyquist $\mathrm{OOK}$ format achieved the best transmission performance, as it required the lowest ROP. For the B2B case, the improvement offered by the adaptive loading was not significant, as the dominant factor was the nonlinearity of the overall system rather than the bandwidth-induced power fading. After transmission in the 2-km long SSMF, however, the CD effect imposed severe power fading to high-frequency signal components. Therefore, the ROP deterioration was much smaller in the adaptively-loaded DDO-OFDM, which can partially mitigate such effects, than in the uniformly-loaded 4QAM DDO-OFDM
Similarly, for $100-\mathrm{Gb} / \mathrm{s}$ transmission, the uniformly-loaded 16QAM DDO-OFDM required the highest ROPs to fulfil the BER requirement. By using adaptive loading, more than 2-dB ROP sensitivity improvement can be obtained compared to the uniformly-loaded case. However, the best transmission performance was still achieved by the single-carrier Nyquist PAM4 format.

TABLE I

COMPARISON OF REQUIRED ROPS FOR 50-GB/S TRANSMISSION

\begin{tabular}{ccccc}
\hline \hline Format & \multicolumn{2}{c}{ B2B } & \multicolumn{2}{c}{ 2-km SSMF } \\
\cline { 2 - 5 }$(50 \mathrm{~Gb} / \mathrm{s})$ & single & diff. & single & diff. \\
\hline Nyquist OOK & $-7.5 \mathrm{dBm}$ & $-9 \mathrm{dBm}$ & $-6 \mathrm{dBm}$ & $-7.5 \mathrm{dBm}$ \\
4 QAM based & $-3.7 \mathrm{dBm}$ & $-5.4 \mathrm{dBm}$ & $-0.8 \mathrm{dBm}$ & $-2.8 \mathrm{dBm}$ \\
$\begin{array}{c}\text { DDO-OFDM } \\
\text { daptively-loaded } \\
\text { DDO-OFDM }\end{array}$ & $-4 \mathrm{dBm}$ & $-5.8 \mathrm{dBm}$ & $-2.7 \mathrm{dBm}$ & $-4 \mathrm{dBm}$ \\
\hline \hline
\end{tabular}

TABLE II

COMPARISON OF REQUIRED ROPS FOR 100-GB/S TRANSMISSION

\begin{tabular}{ccccc}
\hline \hline \multirow{2}{*}{$\begin{array}{c}\text { Format } \\
(100 \mathrm{~Gb} / \mathrm{s})\end{array}$} & \multicolumn{2}{c}{ B2B } & \multicolumn{2}{c}{ 2-km SSMF } \\
\cline { 2 - 5 } Ningle & diff. & single & diff. \\
\hline $\begin{array}{c}\text { Nyquist PAM4 } \\
\text { 16QAM based }\end{array}$ & $-1.6 \mathrm{dBm}$ & $-4 \mathrm{dBm}$ & $0.1 \mathrm{dBm}$ & $-2 \mathrm{dBm}$ \\
$\begin{array}{c}\text { DDO-OFDM } \\
\text { Adaptively-loaded } \\
\text { DDO-OFDM }\end{array}$ & $3.3 \mathrm{dBm}$ & $0 \mathrm{dBm}$ & $>4 \mathrm{dBm}$ & $2.8 \mathrm{dBm}$ \\
\hline \hline
\end{tabular}

\section{Capacity maximization using adaptively-loaded DDO-OFDM}

As mentioned in Section II, the transimpedance gain of the fully-differential TIA can be easily adjusted by changing the voltage supply to the NMOS transistor, i.e., $V_{c t}$. In principle, the lower the $V_{c t}$, the higher the gain of the TIA. However, this will result in a narrower electrical bandwidth of the integrated receiver. Consequently, there will be an optimal setting of $V_{c t}$ to balance the trade-off between the transimpedance gain and the electrical bandwidth, and thus maximise the transmission rate. Furthermore, since CD induces power fading in the DD system, which also largely affects the bandwidth of the transmission, the optimal setting of $V_{c t}$ also relates to the length of the fibre in the link. In this sub-section, we investigate how the operation of the integrated silicon receiver can be adapted to the conditions imposed by the transmission in order to maximise the capacity of the link. Here, we employed the adaptively-loaded DDO-OFDM to maximise the achievable capacity whilst ensuring a BER below the 7\% FEC limit was maintained. The number of modulated data subcarriers was increased to 247 , which corresponded to an electrical bandwidth of $\sim 33.77 \mathrm{GHz}$. Note that this bandwidth was limited by the cut-off frequency of the DSO used in the experiments.

Figs. 13 and 14 show the maximum capacities and the corresponding BERs of the B2B and 2-km long SSMF links, respectively, under different $V_{c t}$ settings. The ROP was fixed to $4 \mathrm{dBm}$ in all cases. For the B2B case, the optimal $V_{c t}$ was around $1.1 \mathrm{~V}$, at which the maximum capacity of the system could be improved from $\sim 152.99 \mathrm{~Gb} / \mathrm{s}$ when using a single-ended output to $\sim 173.22 \mathrm{~Gb} / \mathrm{s}$ by employing the 
differential output of the TIA. Even though the gain of the TIA was greater at smaller $V_{c t}$ values, the capacity was mainly limited by the narrower electrical bandwidth. In contrast, at higher values of $V_{c t}$, the performance was mainly limited by the smaller TIA gain of the integrated silicon receiver. Nevertheless, for all $V_{c t}$ settings, the capacity enhancement obtained by using the differential output was around $20 \mathrm{~Gb} / \mathrm{s}$, as shown in Fig. 13. Furthermore, in all cases, the corresponding BERs were below the $7 \%$ FEC limit, confirming the effectiveness of the adaptive bit-and-power loading.

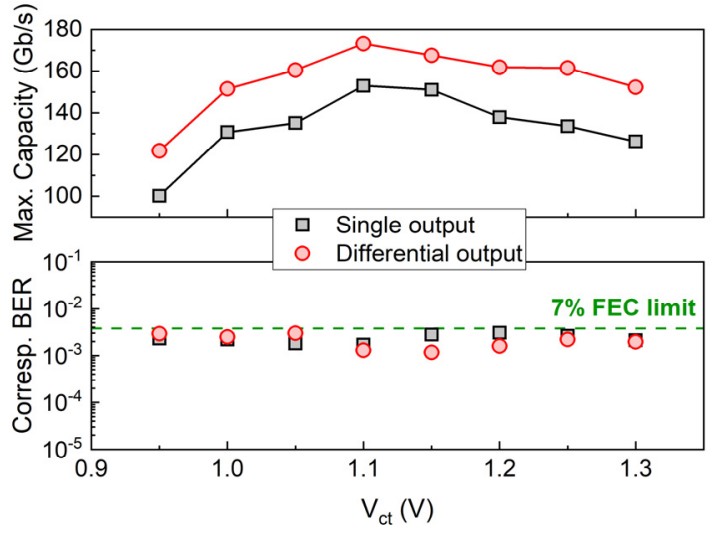

Fig. 13. The maximum capacity versus $V_{c t}$ of the B2B link using the adaptively-loaded DDO-OFDM and the corresponding BER.

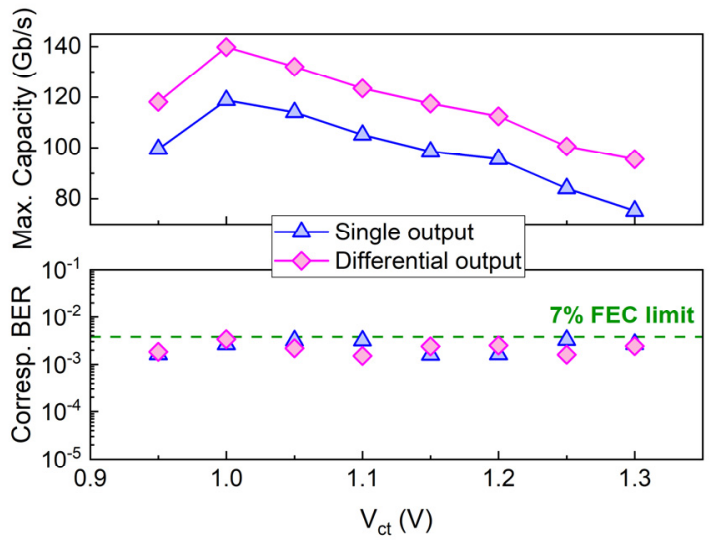

Fig. 14. The maximum capacity versus $V_{c t}$ of the 2-km long SSMF link using the adaptively-loaded DDO-OFDM and the corresponding BER.

The capacity maximization results after transmission in the 2-km long SSMF are shown in Fig. 14. It is seen that the optimal $V_{c t}$ value decreased to $1.0 \mathrm{~V}$ in this case. This is because the usable electrical bandwidth of the overall system is mainly restricted by the CD-induced power fading effect after propagation in the 2-km length of SSMF. Therefore, obtaining a higher TIA gain was more beneficial than ensuring a broader bandwidth. With a $V_{c t}$ of $1.0 \mathrm{~V}$, the maximum capacities of the single-ended and differential output cases were $118.81 \mathrm{~Gb} / \mathrm{s}$ and $139.86 \mathrm{~Gb} / \mathrm{s}$, respectively. As in the B2B case, the capacity enhancement brought by the differential TIA structure was around $20 \mathrm{~Gb} / \mathrm{s}$.

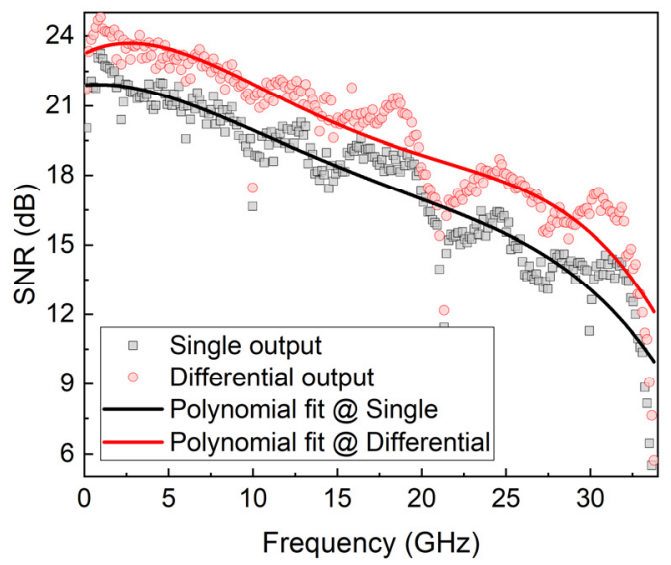

Fig. 15. SNR versus frequency of the B2B link with $V_{c t}=1.1 \mathrm{~V}$.

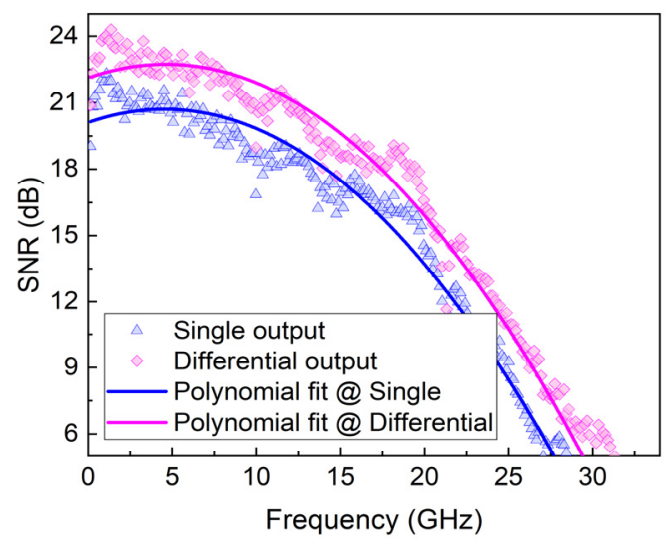

Fig. 16. SNR versus frequency of the 2-km long SSMF link with $V_{c t}=1.0 \mathrm{~V}$.

Figs. 15 and 16 contrast the estimated SNR profiles over the frequency of the B2B and 2-km long SSMF links, respectively, as obtained under the respective optimal $V_{c t}$ setting. A comparison between the two figures highlights the power fading induced spectral narrowing experienced during transmission in the fibre, which supports the previous discussion. Note that the low-SNR spikes in Fig. 15 resulted from clock leakage of the high-speed digital-to-analog converter in the AWG [30], rather than the integrated receiver. Once again, the graphs demonstrate the 2-3-dB SNR improvement that is achieved across the available bandwidth when using the differential output of the receiver, which resulted in an increased capacity. 

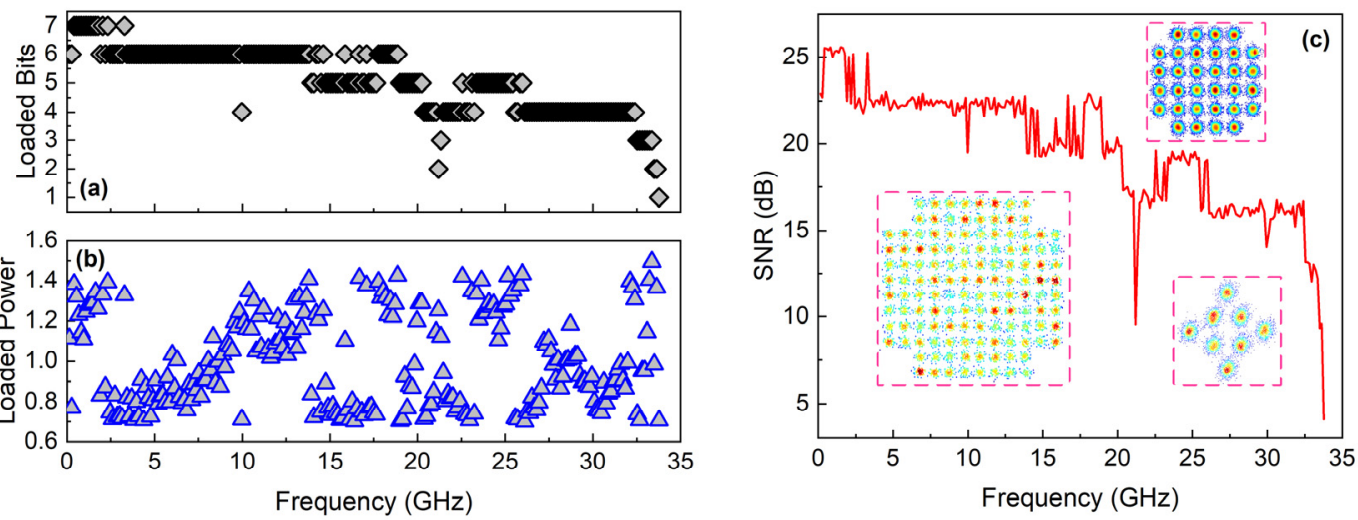

Fig. 17. Results of the adaptively-loaded DDO-OFDM transmission using the differential output in the B2B link with $V_{c t}$ being $1.1 \mathrm{~V}$ : (a) loaded bits, (b) loaded power and (c) estimated SNR after transmission versus frequency. The corresponding capacity was $\sim 173.22 \mathrm{~Gb} / \mathrm{s}$. Insets: some of the recovered constellations.
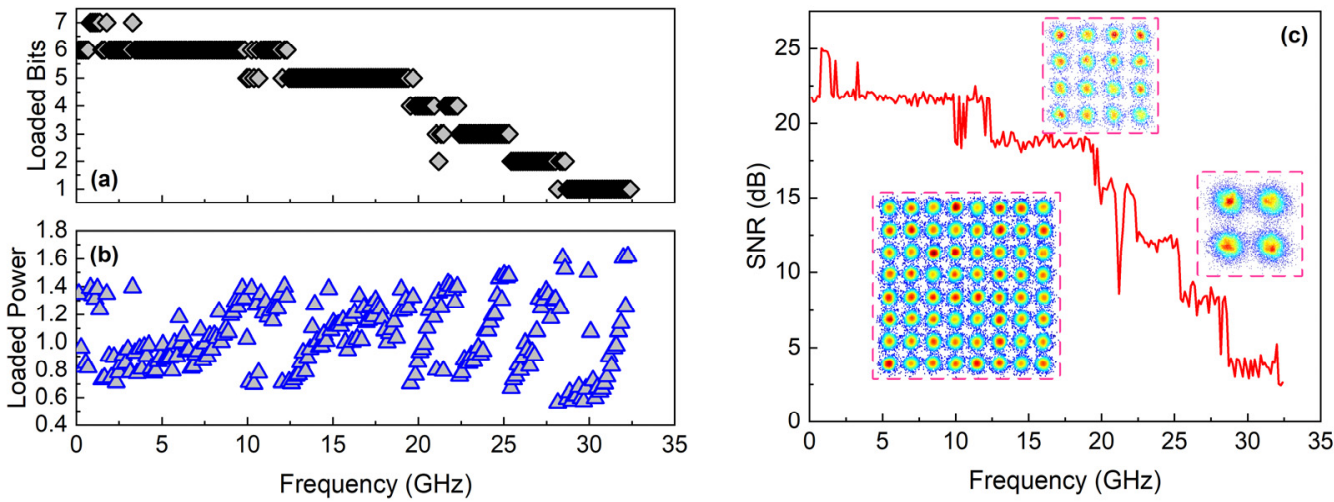

Fig. 18. Results of the adaptively-loaded DDO-OFDM transmission using the differential output in the 2-km long SSMF link with $V_{c t}$ being $1.0 \mathrm{~V}$ : (a) loaded bits, (b) loaded power and (c) estimated SNR after transmission versus frequency. The corresponding capacity was $\sim 139.86 \mathrm{~Gb} / \mathrm{s}$. Insets: some of the recovered constellations.

Figures 17 and 18 show the bit and power loading that was used on the various subcarriers in the cases of the differential receiver output for $\mathrm{B} 2 \mathrm{~B}$ and $2-\mathrm{km}$ long transmission, respectively. These were obtained using the estimated SNR profiles of Figs. 15 and 16 as their bases and show that more bits were allocated to the frequencies with higher SNRs. Considering the low-pass-like property of the frequency response, the power allocation exhibited a saw-tooth profile, so that the BER performance of adjacent subcarriers can be similar. Furthermore, a comparison between Figs.17 and 18 shows clearly how the power fading at high frequencies experienced during transmission restricts the overall capacity of the link. Under these conditions, the capability to tune the balance between TIA gain and receiver bandwidth is very useful for optimising the link performance.

\section{E. Comparison to the State-of-the-Art}

Finally, we compare this work with state-of-the-art transmission demonstrations using TIA-integrated silicon optical receivers, as presented in Table III. It is seen that most of the previous works focused on the characterisation of the fabricated optical receivers, hence only B2B transmission was considered in their tests. Furthermore, in high-speed demonstrations $(>90 \mathrm{~Gb} / \mathrm{s})$, transmitter DSP was used to compensate for the bandwidth limitations of either the AWG or modulator $[16-17,19]$. Other than this, the transmission tests relied on simple OOK/PAM4. Unlike the previous demonstrations, Nyquist shaping was adopted in this work to halve the signal bandwidth of the OOK/PAM4 format. Although Nyquist shaping increases the peak-to-average power ratio of the resulting time-domain signal, the good linearity performance of the fabricated optical receiver allowed successful transmission of 100-Gb/s Nyquist PAM4 signals in both B2B and the 2-km long SSMF links. Moreover, by using adaptive loading in multi-carrier DDO-OFDM transmission, we achieved the highest data rate $(\sim 173.22 \mathrm{~Gb} / \mathrm{s}$ in $\mathrm{B} 2 \mathrm{~B})$ amongst the works appearing in the table. Even after 2-km long transmission, a data rate of $\sim 139.86 \mathrm{~Gb} / \mathrm{s}$ was achieved whilst keeping the BER below the $7 \%$ FEC limit.

\section{CONCLUSIONS}

In this paper, we extensively investigated the performance of transmission systems using a silicon optical receiver which integrated a SiGe balanced PD and a fully-differential 28-nm CMOS TIA. Various modulation formats, including single-carrier Nyquist OOK/PAM4 and multi-carrier DDO-OFDM, were considered to perform a comparative study that tested the linearity performance of the integrated receiver module. It was shown that for $50-\mathrm{Gb} / \mathrm{s}$ and $100-\mathrm{Gb} / \mathrm{s}$ 
TABLE III

COMPARISON WITH THE STATE-OF-THE-ART DEMONSTRATIONS

\begin{tabular}{|c|c|c|c|c|c|c|}
\hline Ref. & Technology & TIA Design & Format & Data Rate & Reach & DSP \\
\hline$[16]$ & 55-nm BiCMOS & Diff. & OOK & 90Gb/s@10 $0^{-5}$ & B2B & Tx 6-tap FFE \\
\hline [17] & 55-nm BiCMOS & Diff. & PAM4 & 106Gb/s@2.09×10-4 & $\mathrm{B} 2 \mathrm{~B}$ & AWG equalization \\
\hline [18] & 65-nm CMOS & Diff. & OOK & $32 \mathrm{~Gb} / \mathrm{s}$ & B2B & Nil \\
\hline [19] & 28-nm CMOS & S2D & PAM4 & $112 \mathrm{~Gb} / \mathrm{s} @<10^{-4}$ & $\mathrm{~B} 2 \mathrm{~B}$ & Tx 3-tap FFE \\
\hline [21] & 28-nm CMOS & Single & OOK & $53 \mathrm{~Gb} / \mathrm{s} @<10^{-12}$ & B2B & Nil \\
\hline This work & 28-nm CMOS & Diff. & $\begin{array}{c}\text { Nyquist OOK } \\
\text { Nyquist PAM4 }\end{array}$ & $\begin{array}{c}50 \mathrm{~Gb} / \mathrm{s} @ 3.8 \times 10^{-3} \\
100 \mathrm{~Gb} / \mathrm{s} @ 3.8 \times 10^{-3}\end{array}$ & B2B\&2-km & $\mathrm{Rx}(17,7)$-tap DFE \\
\hline This work & 28-nm CMOS & Diff. & DDO-OFDM & $\begin{array}{l}173.22 \mathrm{~Gb} / \mathrm{s} @ 3.8 \times 10^{-3} \\
139.86 \mathrm{~Gb} / \mathrm{s} @ 3.8 \times 10^{-3}\end{array}$ & $\begin{array}{c}\text { B2B } \\
\text { 2-km SMF }\end{array}$ & Adaptive loading \\
\hline
\end{tabular}

Note: 'Diff.' stands for 'Differential' and 'S2D' denotes 'Single-to-Differential'.

transmission in both $\mathrm{B} 2 \mathrm{~B}$ and 2-km long SSMF links, the single-carrier OOK/PAM4 exhibited better transmission performance. A lower ROP was required to achieve a BER below the 7\% FEC limit, compared to that of either the uniformly- or adaptively-loaded DDO-OFDM formats. In addition, it was validated that the differential TIA structure is beneficial to all of the modulation formats we considered, offering significant BER and SNR improvements. Moreover, we demonstrated the flexibility offered by the integrated receiver in terms of balancing the transimpedance gain and the electrical bandwidth, which is crucial to maximising the capacity of the overall system after transmission in different lengths of SSMF. With the help of the adaptively-loaded DDO-OFDM and the differental output of the integrated receiver, maximum capacities of $\sim 173.22 \mathrm{~Gb} / \mathrm{s}$ and $\sim 139.86$ $\mathrm{Gb} / \mathrm{s}$ were achieved at a BER limit of $3.8 \times 10^{-3}$ for the B2B and 2-km long SSMF links, respectively. Our results indicate the feasibility of using the standard CMOS-process-based optical receiver to realise $>100-\mathrm{Gb} / \mathrm{s}$ transmission, highlighting its promising potential for low-cost and high-speed DD transmission.

\section{ACKNOWLEDGMENT}

The data for this work is accessible through the University of Southampton Institutional Research Repository (DOI: https://doi.org/10.5258/SOTON/D1567).

\section{REFERENCES}

[1] E. Agrell, M. Karlsson, A.R. Chraplyvy, et al., "Roadmap of Optical Communications," J. Opt., vol. 18, pp. 1-40, 2016.

[2] Q. Cheng, M. Bahadori, M. Glick, et al., "Recent Advances in Optical Technologies for Data Centers: A Review," Optica, vol. 5, no. 11, pp. 1354-1370, 2018.

[3] J. Cheng, C. Xie, Y. Chen, et al., "Comparison of Coherent and IM/DD Transceivers for Intra Datacenter Optical Interconnects," in Proc. of OFC, San Diego, USA, paper W1F.2, 2019.

[4] R. Urata, H. Liu, X. Zhou, et al., "Data center Interconnect and Networking: from Evolution to Holistic Revolution," in Proc. of $O F C$, Los Angeles, USA, paper W3G.1, 2017

[5] IEEE P802.3bs. Accessed: Aug. 05, 2019. [Online]. Available: $\mathrm{http}: / / \mathrm{www}$. ieee802.org/3/bs/

[6] 100G Lambda Multi-Source Agreement. Accessed: Aug. 05, 2019. [Online]. Available: http://100glambda.com

[7] V. Stojanović, R. J. Ram, M. Popović, et al., "Monolithic silicon-photonic platforms in state-of-the-art CMOS SOI processes," Opt. Express, vol. 26, no. 10, pp. 13106-13121, 2018.

[8] D. J. Thomson, K. Debnath, W. Zhang, et al., "Towards High Speed and Low Power Silicon Photonic Data Links," in Proc. of ICTON, Bucharest, Romania, paper Th.A.3.3, 2018.
[9] K. Zhong, X. Zhou, J. Huo, et al., "Digital Signal Processing for Short-Reach Optical Communications: A Review of Current Technologies and Future Trends," J. Light. Technol., vol. 36, no. 2, pp. 377-400, 2018.

[10] X. Pang, O. Ozolins, L. Zhang, et al., "Beyond 200 Gbps per Lane Intensity Modulation Direct Detection (IM/DD) Transmissions for Optical Interconnects: Challenges and Recent Developments," in Proc. of $O F C$, San Diego, USA, paper W4I.7, 2019.

[11] P.D. Heyn, V.I. Kopp, S.A. Srinivasan, et al., "Ultra-dense $16 \times 56 \mathrm{~Gb} / \mathrm{s}$ NRZ GeSi EAM-PD Arrays Coupled to Multicore Fiber for Short-reach 896Gb/s Optical links," in Proc. of OFC, Los Angeles, USA, paper Th1B.7, 2017.

[12] J. Verbist, M. Verplaetse, S. A. Srinivasan, et al., "Real-Time $100 \mathrm{~Gb} / \mathrm{s}$ NRZ and EDB Transmission With a GeSi Electroabsorption Modulator for Short-Reach Optical Interconnects," J. Light. Technol., vol. 36, no. 1, pp. 90-96, 2018.

[13] B. Moeneclaey, G. Kanakis, J. Verbrugghe, et al., "A 64 Gb/s PAM-4 Linear Optical Receiver," in Proc. of OFC, Los Angeles, USA, paper M3C.5, 2015.

[14] D. Li, G. Minoia, M. Repossi, et al., "A 25Gb/s 3D-integrated silicon photonics receiver in $65 \mathrm{~nm}$ CMOS and PIC25G for $100 \mathrm{GbE}$ optical links," in Proc. of ISCAS, Montreal, Canada, pp. 2334-2337, 2016.

[15] I. G. López, A. Awny, P. Rito, et al., "100 Gb/s Differential Linear TIAs With Less Than $10 \mathrm{pA} / \sqrt{ } \mathrm{Hz}$ in 130-nm SiGe:C BiCMOS," J. Light. Technol., vol. 53, no. 2, pp. 458-469, 2018.

[16] J. Lambrecht, H. Ramon, B. Moeneclaey, et al., "90-Gb/s NRZ Optical Receiver in Silicon Using a Fully Differential Transimpedance Amplifier," J. Light. Technol., vol. 37, no. 9, pp. 1964-1973, 2019.

[17] J. Lambrecht, H. Ramon, B. Moeneclaey, et al., "A 106-Gb/s PAM-4 Silicon Optical Receiver," IEEE Photon. Technol. Lett., vol. 31, no. 7, pp. 505-508. 2019.

[18] S. G. Kim, C. Hong, Y. S. Eo, et al., "A 40-GHz Mirrored-Cascode Differential Transimpedance Amplifier in 65-nm CMOS," IEEE J. Solid-State Circuits, vol. 54, no. 5, pp. 1468-1474, 2019.

[19] H. Li, G. Balamurugan, J. Jaussi, et al., "A 112 Gb/s PAM4 Linear TIA with $0.96 \mathrm{pJ} / \mathrm{bit}$ Energy Efficiency in $28 \mathrm{~nm}$ CMOS," in Proc. of ESSCIRC, Dresden, Germany, pp. 238-241, 2018.

[20] Y. Hong, K. Li, C. Lacava, et al., "Beyond 100-Gb/s Direct-detection Transmission using an Optical Receiver Co-integrated with a 28-nm CMOS Gain-tunable Fully-differential TIA," in Proc. of OFC, San Diego, USA, paper Th3J.4, 2020.

[21] L. Szilagyi, J. Pliva, R. Henker, et al., "A 53-Gbit/s Optical Receiver Frontend with $0.65 \mathrm{pJ} / \mathrm{bit}$ in $28-\mathrm{nm}$ Bulk-CMOS," IEEE J. Solid-State Circuits, vol. 54, no. 3, pp. 845-854, 2019.

[22] K. Li, S. Liu, X. Ruan, et al., "Co-design of Differential Transimpedance Amplifier and Balanced Photodetector for Sub-pJ/bit Silicon Photonics Receiver," Opt. Express, vol. 28, no. 9, pp. 14038-14054, 2020.

[23] P. Chevalier, W. Liebl, H. RÜcker, et al., "SiGe BiCMOS Current Status and Future Trends in Europe," in Proc. of BCICTS, San Diego, USA, pp. 64-71, 2018

[24] A. H. Pawlikiewicz, and David Hess, "Choosing RF CMOS or SiGe BiCMOS in mixed-signal design," Accessed: Aug. 05, 2019. [Online]. Available:

https://pdfs.semanticscholar.org/1282/6ad38819ef80808e418d73ea12b2 ef631224.pdf

[25] O. Ishida, K. Takei, and E. Yamazaki, "Power Efficient DSP Implementation for $100 \mathrm{G}$-and-beyond Multi-haul Coherent Fiber-optic Communications," in Proc. of OFC, paper W3G.3, 2016. 
[26] G. Muliuk, K. V. Gasse, J. V. Kerrebrouck, et al., " $4 \times 25$ Gbps Polarization Diversity Silicon Photonics Receiver With Transfer Printed III-V Photodiodes," IEEE Photon. Technol. Lett., vol. 31, no. 4, pp. 287-290, 2019.

[27] M. Ma, K. Murray, M. Ye, et al., "Silicon Photonic Polarization Receiver with Automated Stabilization for Arbitrary Input Polarizations," in Proc. of CLEO, San Jose, USA, paper STu4G.8, 2016.

[28] P. S. Chow, J. M. Cioffi, and J. A. C. Bingham, "A Practical Discrete Multitone Transceiver Loading Algorithm for Data Transmission over
Spectrally Shaped Channels," IEEE Trans. on Commun., vol. 73, no. 2 , pp. 773-775, 1995.

[29] Y. Hong, K. R. H. Bottrill, N. Taengnoi, et al. "Beyond $100-\mathrm{Gb} / \mathrm{s} / \lambda$ Direct-detection Transmission over the $\mathrm{S}+\mathrm{C}+\mathrm{L}-$ bands in An Ultra-wide Bandwidth Hollow Core Fibre," in Proc. of ECOC, Dublin, Ireland, paper Th.2.E.5, 2019

[30] X. Ouyang, G. Talli, M. Power, et al., "Orthogonal Chirp-division Multiplexing for IM/DD-based Short-reach Systems," Opt. Express, vol. 27, no. 16, pp. 23620-23632, 2019. 